\title{
Valoración radiológica a los tres y cinco años de la pérdida y calidad ósea periimplantaria en implantes Branemark
}

\author{
DONADO AZCÁRATE A * \\ PERIS GARCÍA-PATRÓN R $\mathbf{R}^{\mathrm{a}} \mathbf{M}$ ** \\ LÓPEZ-QUILES MARTÍNEZ, J *** \\ SADA GARCÍA-LOMAS JM $* * * *$
}

\author{
Donado Azcárate A, Peris García-Patron RaM, López-Quiles \\ Martínez J, Sada García-Lomas JM. Valoración radiológica a \\ los tres y cinco años de la pérdida y calidad ósea \\ periimplantaria en implantes Branemark. Av Periodon \\ Implantol. 2001; 13, 1: 19-27
}

\begin{abstract}
RESUMEN
OBJETIVOS:Valorar la reabsorción marginal y la calidad ósea en torno a implantes Branemark comparando entre sí ambos parámetros a los tres y cinco años de función.

MATERIAL Y MÉTODO: Se estudiaron 139 implantes Branemark realizados en el Máster de Implantes de la Facultad de Odontología de Madrid colocados por profesores del Departamento y que soportaban exclusivamente prótesis fijas, y se valoró mediante estudio radiográfico estandarizado la reabsorción y la calidad ósea en torno a los implantes

RESULTADOS: Si bien a los tres años no puede establecese una relación entre la pérdida marginal y la calidad ósea periimplantaria, se comprueba estadísticamente que aquellos implantes que a los tres años demostraron una mejor calidad a los cinco sufrieron una menor reabsorción; igualmente los implantes que en el primer control sufrieron menor pérdida, en el segundo poseían una mejor calidad y, finalmente, a los cinco años siempre fueron coincidentes los implantes con menor reabsorción y mejor calidad.

CONCLUSIONES: En nuestra muestra resulta evidente la relación estadística entre los parámetros estudiados pero ello no nos permite hacer un pronóstico a largo plazo con los resultados observados a los 3 años. Si bien podemos decir que una buena calidad ósea en los primeros controles parece garantizarnos menor reabsorción en el futuro, no podemos asegurar que una peor calidad augure el fracaso del tratamiento.
\end{abstract}

\section{PALABRAS CLAVE}

Reabsorción ósea periimplantaria; calidad ósea periimplantaria.

\section{INTRODUCCIÓN}

En la actualidad es generalmente aceptado por la comunidad científica que es necesario un período mínimo de 5 años en funcionamiento de la prótesis implantosoportada para poder establecer que el tratamiento ha sido realizado con éxito ( criterios de éxito de Albrektsson, Zarb, Whortington y Ericksson

* Odontólogo. Prof. Ayudante del Departamento de Medicina y Cirugía Bucofacial. Facultad de Odontología. Universidad Complutense de Madrid.

** Odontólogo. Prof. Asociado del Departamento de Medicina y Cirugía Bucofacial. Facultad de Odontología. Universidad Complutense de Madrid.

*** Médico Estomatólogo. Prof. Asociado del Departamento de Medicina y Cirugía Bucofacial. Facultad de Odontología. Universidad Complutense de Madrid.

**** Cirujano Maxilofacial. M. Estomatólogo. Prof. Titular del Departamento de Medicina y Cirugía Bucofacial. Facultad de Odontología. Universidad Complutense de Madrid. 
en 1986 (1). Pero es también confirmado por la experiencia clínica y por los estudios realizados por autores de la talla de Adell $(2,3)$ que la mayor parte de la pérdida de fijaciones se produce durante los tres primeros años a partir de la colocación de las mismas, y que la mayor reabsorción ósea marginal ocurre durante el primer año de funcionamiento de la prótesis, para disminuir considerablemente y hacerse estable en los años siguientes. Asimismo, el mayor número de fijaciones no osteointegradas acontecen durante este período de tiempo.

Partiendo pues de estas últimas premisas, y basándonos en la hipótesis, de que todos aquellos pacientes portadores de prótesis fija implantosoportadas en funcionamiento desde al menos hace tres años cumplieran los criterios establecidos como de éxito, podría establecerse que no excedería más allá de 36 meses el tiempo de seguimiento de la prótesis, necesario para garantizar a largo plazo el éxito de la misma, ya que habiéndose producido la mayor pérdida marginal durante el primer año, serían suficientes sólo dos años más para comprobar que la reabsorción ha sido estable según lo comprobado en otros estudios. Al comparar los resultados obtenidos en la revisión a los 3 años con otra realizada a los 5 años tal y como se establece en los criterios de éxito, podremos afirmar si los implantes de una muestra se encuentran en una situación controlada desde los 3 años o si por el contrario pueden esperarse alteraciones en los parámetros de seguimiento.

\section{OBJETIVOS}

Los objetivos que pretendemos alcanzar con el presente estudio son los siguientes:

- Valorar la reabsorción marginal y calidad ósea periimplantaria de las fijaciones en funcionamiento desde hace 36 meses o más.

Relacionar entre sí clinica y estadísticamente ambos parámetros.

\section{MATERIAL Y MÉTODO}

\section{MATERIAL HUMANO}

El estudio que se desarrolla en este trabajo ha sido realizado sobre pacientes parcial o totalmente desdentados tratados por miembros del equipo de implantología del Máster de Implantes de la Facultad de Odontología de Madrid con 139 implantes osteointegrados tipo Branemark y que han sido posteriormente portadores de prótesis fijas.

\section{Material para la exploración radiográfica}

Radiografías periapicales Kodak DF 57 Ultra Speed

Tubo de rayos X Ritter Transdent 502 con un tiempo de exposición 0'1-0'4 y valores de $12 \mathrm{~mA}$ y 70KV

- Sistema Porta-Rinn para estandarización de proyecciones

- Lupa de 10 aumentos

- Negatoscopio tipo Rex, 220v/50HV-GMBH

- Regla milimetrada

- Compás de puntas milimetrado

a Procesador Philiphs 9000.

\section{MÉTODO}

Realización de radiografías periapicales de todas las fijaciones mediante el sistema Porta-Rinn $(4,5,6,7)$. Para ello se coloca la película de forma ortorradial a la fijación respectiva, con sus bordes paralelos al eje longitudinal de la misma. La zona de imagen ha de extenderse por lo menos $5 \mathrm{~mm}$. dentro del hueso a ambos lados del implante hacia las fijaciones vecinas. Las estrías del implante deben aparecer nítidamente en la radiografía de tal forma que pueda examinarse la unión con el tejido óseo en el fondo de rosca (Fig.1). Es imprescindible que la placa se mantenga plana en el porta-film.

Mediante las radiografías periapicales realizadas durante la fase clínica del estudio pudo comprobarse la pérdida ósea marginal ocurrida durante el tiempo que llevó la prótesis en funcionamiento al establecerse una comparación con las tomadas después de la

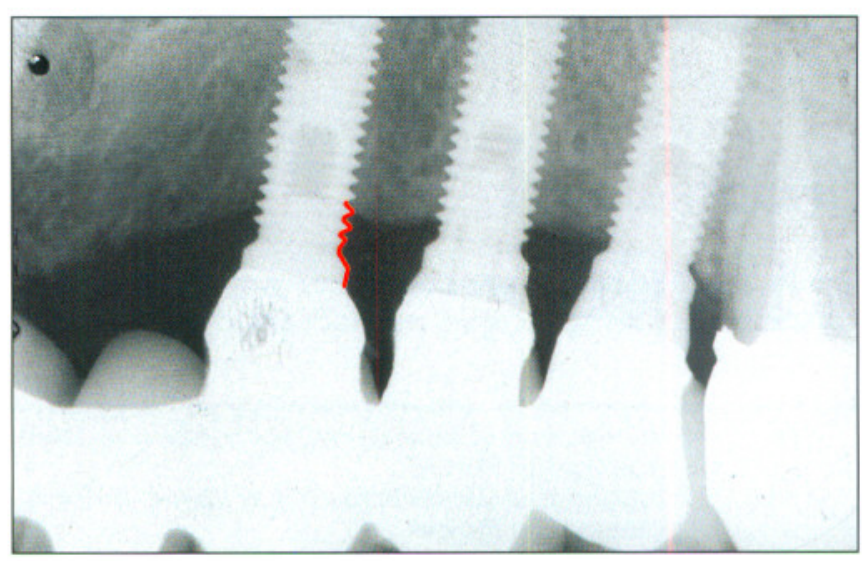

Figura 1. 


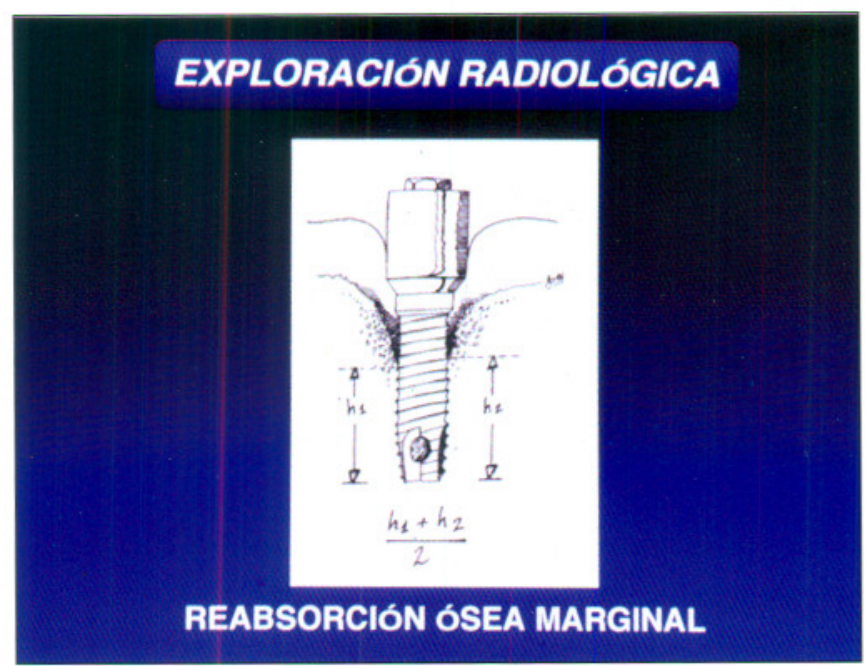

Figura 2.

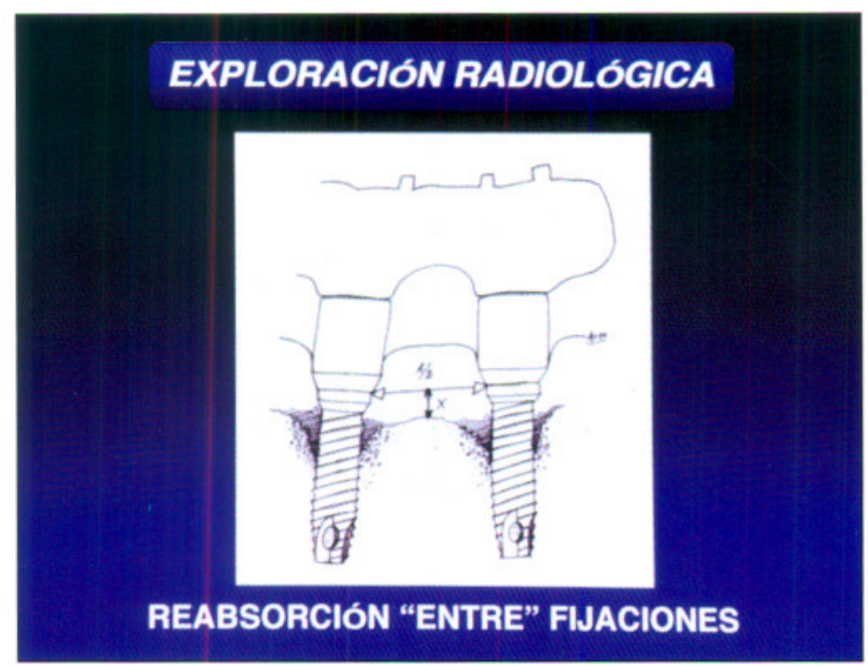

Figura 3.

colocación de los implantes tras la fase de reposo. La realización de las radiografías fue llevada a cabo por el mismo investigador, de tal forma que existieran las menores diferencias posibles en el conjunto de todo el estudio radiográfico.

Cada una de las mediciones fue realizada por 3 investigadores diferentes de forma que se obtuvo la media de todas. Si una medición discrepaba en $0,4 \mathrm{~mm}$. o más no se tenía en cuenta y se realizaba la media con los otros dos valores (Hollender y Rockler)(5).

El análisis de los parámetros mencionados se estableció según la siguiente metodología:

\section{Pérdida ósea marginal en torno a las fijaciones:}

Se midió en la radiografía periapical la distancia tanto

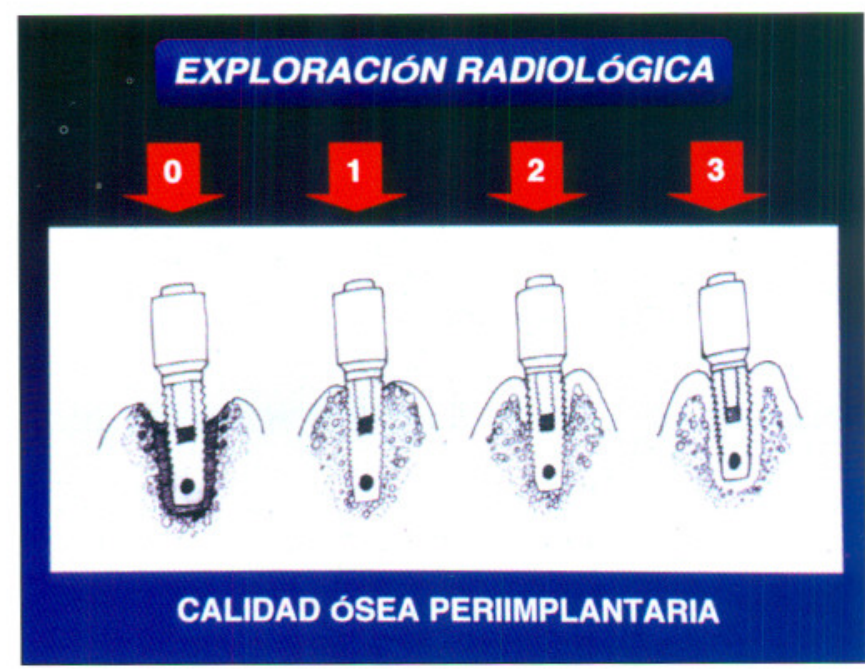

Figura 4.

en mesial como en distal desde un punto de referencia tomado en la fijación ( zona de unión pilar-implante ) hasta la zona de contacto entre el titanio y el tejido óseo $(8,9)$. No se tuvo en cuenta aquellos puntos en los que se encontraba en contacto el hueso con el pilar, ya que se considera que en dicha zona no existe osteointegración. Mediante la fórmula( $1 / 2(\mathrm{hl}+\mathrm{h} 2)$ ) obtenemos la media de dicha pérdida en torno a la fijación, siendo hl la pérdida producida en mesial y h2 la producida en distal a la fijación (10) (Fig.2 ). Como referencia interna se utilizó la medida de $0,6 \mathrm{~mm}$. entre dos espiras del implante.

\section{Pérdida ósea entre fijaciones:}

Se midió la longitud de una línea imaginaria perpendicular en su mitad a la línea de unión entre dos puntos de referencia prefijados en los implantes y que llegue hasta el nivel óseo. Este punto de referencia es la unión entre la fijación y el pilar. En las fijaciones distales hubo que tomar otro punto de referencia para trazar dicha línea (perpendicular que delimite el borde posterior de la prótesis ) (8,9) (Fig.3).

\section{Calidad ósea periimplantaria:}

Se midió la calidad del tejido óseo de soporte que rodea al implante, según la siguiente tabla: nivel $1=$ hueso trabecular en torno a la fijación, nivel 2 =hueso radiopaco marginal con hueso trabeculado en zonas más profundas, nivel 3 =hueso compacto en todo el entorno del implante (8,9) (Fig.4).

ESTUDIO ESTADÍSTICO ( ANÁLISIS DE RESULTADOS) El estudio estadístico fue realizado en el Centro de Proceso de Datos de la UCM en el seno del área de 
Apoyo a la Investigación.El Software estadístico utilizado ha sido el BMDP ( Dixon WJ, 1993. BMDP Statistical Software Manual. University of California Press).

\section{RESULTADOS}

\section{ANÁLISIS DESCRIPTIVO DE LOS PARÁMETROS EXPLORADOS}

La reabsorción ósea marginal periimplantaria media observada a los tres y cinco años se distribuyó de la siguiente manera (3 años: $m$ 0,077 sd 0,121) (5 años: $m$ 0,106 sd 0,128):

\begin{tabular}{|c|c|c|}
\hline Reabsorc.(mm.) & $\begin{array}{c}\mathbf{3} \text { años (\%) } \\
(\mathbf{0 , 0 7 7 + / - 0 , 1 2 1 )}\end{array}$ & $\begin{array}{c}\mathbf{5} \text { años } \mathbf{( \% )} \\
\mathbf{( 0 , 1 0 6 + / - 0 , 1 2 8 )}\end{array}$ \\
\hline $0-1$ & 67,6 & 61,3 \\
\hline $1-2$ & 14,4 & 6,5 \\
\hline $2-3$ & 13 & 19,3 \\
\hline $3-4$ & 0,7 & 9,7 \\
\hline $4-5$ & 2,1 & 3,2 \\
\hline $5-6$ & 1,4 & 0 \\
\hline $7-8$ & 0,7 & 0 \\
\hline
\end{tabular}

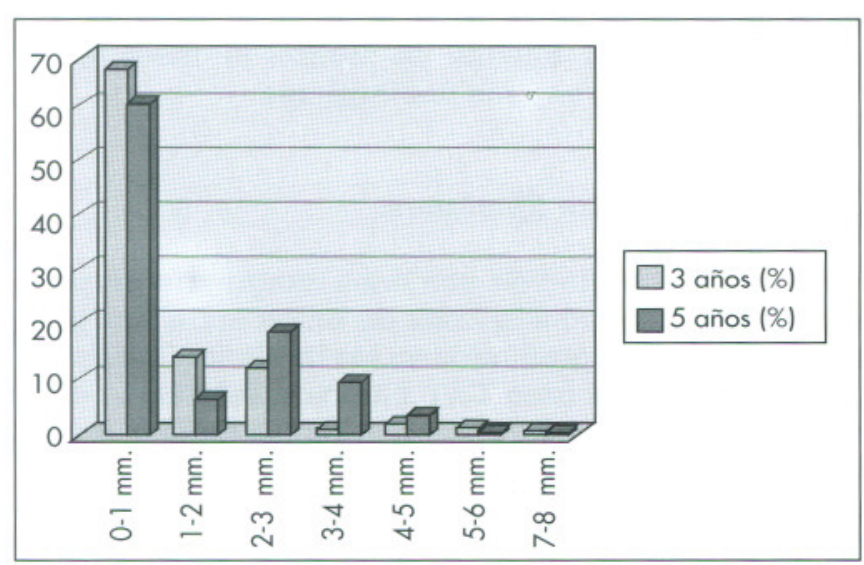

La reabsorción ósea entre los implantes medida a los tres y cinco años (105 localizaciones ), (3 años: m 0,04 sd 0,099 ), (5 años: $m$ 0,111 sd 0,172):

\begin{tabular}{|c|c|c|}
\hline $\begin{array}{c}\text { Reabsorc. } \\
\text { "Entre" (mm) }\end{array}$ & $\begin{array}{c}3 \text { años (\%) } \\
(\mathbf{0 , 0 4 + / - 0 , 0 9 9 )}\end{array}$ & $\begin{array}{c}\mathbf{5} \text { años (\%) } \\
(\mathbf{0 , 1 1 1 + / - 0 , 1 7 2 )}\end{array}$ \\
\hline $0-1$ & 79,0 & 68,2 \\
\hline $1-2$ & 10,5 & 0 \\
\hline $2-3$ & 5,8 & 9,1 \\
\hline $3-4$ & 1,0 & 13,6 \\
\hline $4-5$ & 3,8 & 9,1 \\
\hline
\end{tabular}

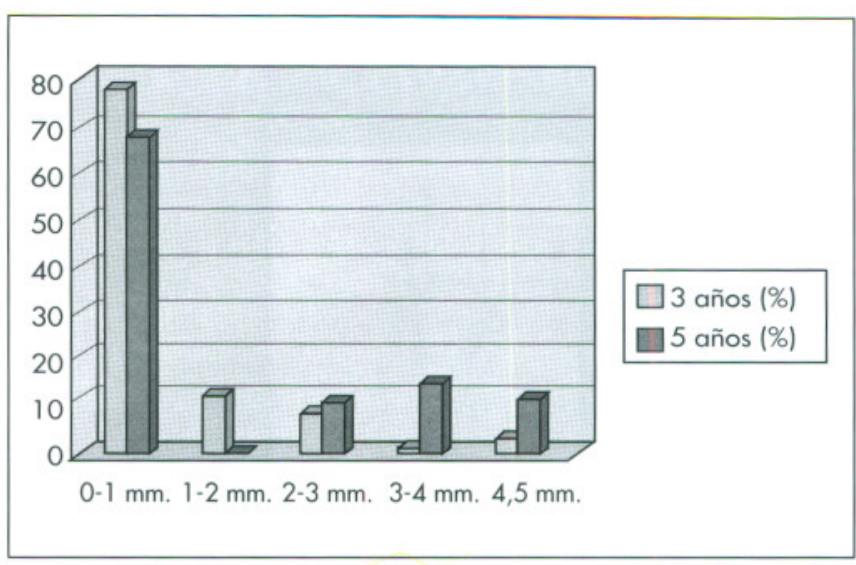

También se valoró la calidad ósea en torno a los implantes según la escala comentada en la metodología. Dicha valoración realizada a los 3 años ofreció el siguiente resultado (m 1,396 sd 0,677):

Calidad ósea grado 0: en el 0,7\% de los IOI. Calidad ósea grado 1: en el 69,1\% de los IOI. Calidad ósea grado 2 : en el $20,1 \%$ de los IOI. Calidad ósea grado 3 : en el 10,1 \% de los IOI.

- A los 5 años, la calidad ósea periimplantaria fue (ml,839 sd 0,820):

Calidad ósea grado 1: en el 41,9\% de los IOI. Calidad ósea grado 2 : en el 32,3\% de los IOI. Calidad ósea grado 3 : en el $25,8 \%$ de los IOI.

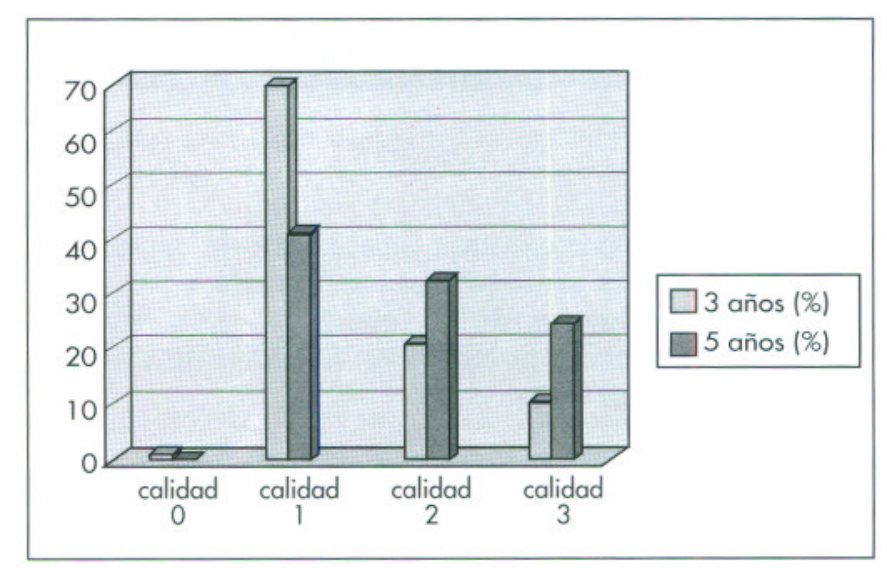

\section{CORRELACIONES ENTRE LOS DIFERENTES PARÁMETROS}

Relación entre la reabsorción ósea marginal y la calidad ósea periimplantaria: 
En las mediciones realizadas a los 3 años de función de los implantes, no se encuentra relación alguna entre el grado de reabsorción marginal en torno a los mismos y la calidad del hueso que los rodea $(\mathrm{p}=0,621)$.

En cambio sí se establece una relación entre la calidad periimplantaria valorada a los 3 años y la rabsorción que posteriormente se produce en estos mismos implantes a los 5 años. De tal forma que aquellas fijaciones que tienen una mejor calidad ósea en la primera medición, han sufrido posteriormente una menor reabsorción de ese hueso $(p=0,040)$. Es decir, hay una asociación significativa entre valores altos de calidad ósea a los 3 años y bajos de reabsorción a los 5 años. Esta correlación se establece a través de la siguiente ecuación:

$\mathrm{Y}=0,21548-0,06676 \cdot \mathrm{X}$. Siendo $\mathrm{Y}=$ reabsorción ósea 5 años (m 0,10565 sd 0,12759) y $\mathrm{X}=$ calidad ósea 3 años (m 1,6452 sd 0,70938).

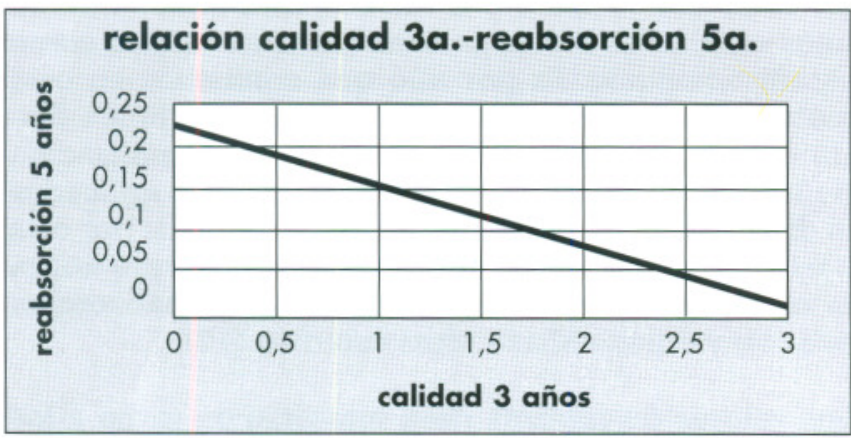

Asimismo, si comparamos la reabsorción ósea producida a los 3 años y la calidad ósea de estas localizaciones a los 5, se comprueba que precisamente aquellos implantes que han sufrido una menor reabsorción en el primer período son los que presentan una mejor calidad en el segundo $(p=0,012)$. $\mathrm{Y}=0,16812-0,05222 \cdot \mathrm{X}$.

$\mathrm{Y}=$ reabsorción ósea 3 años (m 0,07210 sd 0,09573).

$\mathrm{X}=$ calidad ósea 5 años (m 1,8387 sd 0,09573)

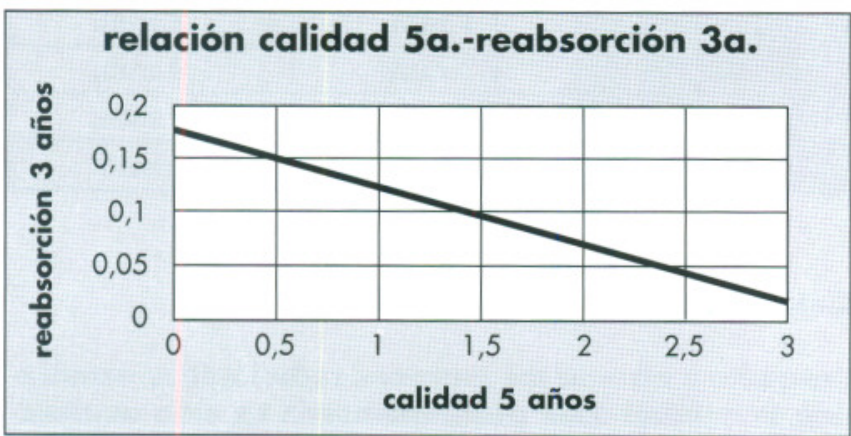

También se verifica estadísticamente que no sólo existe una correlación entre la calidad ósea a los 5 años y la reabsorción a los 3 , sino que ésta también se produce entre los dos parámetros medidos en el segundo período. Es decir, para aquellas localizaciones periimplantarias que tienen una mejor calidad ósea a los 5 años se ha producido también una menor reabsorción $(\mathrm{p}=0,016)$. $\mathrm{Y}=0,22827$ $0,06669 \cdot \mathrm{X}$.

$\mathrm{Y}=$ reabsoción ósea 5 años (m 0,10565 sd 0,12759).

$\mathrm{X}=$ calidad ósea 5 años (m 1,8387 sd 0,82044).

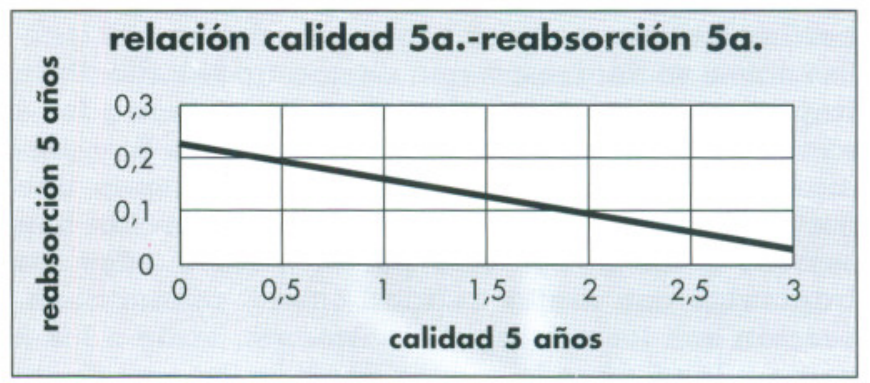

Finalmente si establecemos una correlación entre la reabsorción ósea a los 3 años en cada una de las localizaciones y ese mismo parámetro a los 5 años, se observa que aquellos implantes que han sufrido menor pérdida marginal a los 3 años son también los que presentan valores más bajos a los $5(p=0,001)$. $Y=$ $0,02241+1,1546 \cdot \mathrm{X}$.

$\mathrm{Y}=$ reabsorción ósea 5 años (m 0,10565 sd 0,12759). $\mathrm{X}=$ reabsorción ósea 3 años (m 0,07210 sd 0,09573).

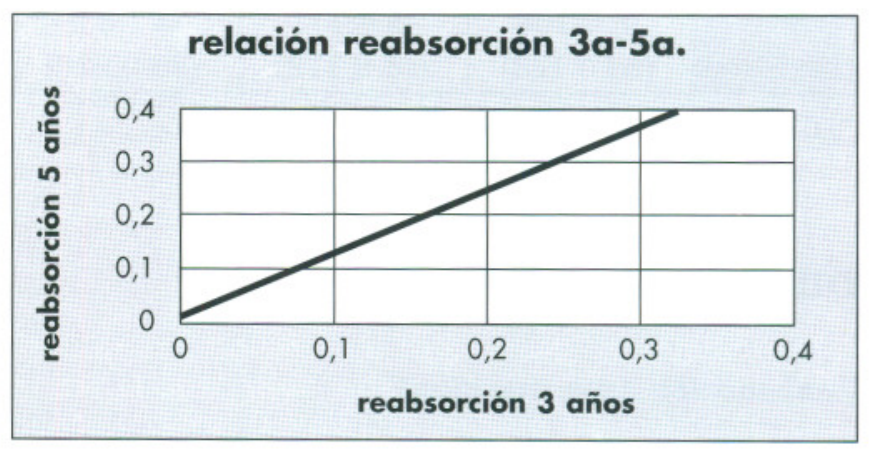

También se han relacionado estas variables con 105 medidas tomadas de la reabsorción ósea sufrida entre las 139 fijaciones estudiadas, ya que en opinión de algunos autores, éstas pueden ser tan indicativas como la medición de la pérdida ósea marginal.

En nuestro estudio se confirma esta teoría ya que hay una correlación directa entre la reabsorción ósea sufrida en los espacios interimplantarios y la reabsorción marginal media de cada fijación tanto a los $3(p=0,001)$ como a los 5 años $(p=0,001)$.

A los 3 años: $\mathrm{Y}=0,05500+0,58773 \cdot \mathrm{X}$

$\mathrm{Y}=$ reabsorción ósea media por implante a los 3 años. $\mathrm{X}=$ reabsorción ósea entre implantes a los 3 años. 
$A$ los 5 años: $\mathrm{Y}=0,04426+0,61484 \cdot \mathrm{X}$

$\mathrm{Y}=$ reabsorción ósea media por implante a los 5 años. $\mathrm{X}=$ reabsorción ósea entre implantes a los 5 años.

Y aunque no las detallamos para no extendernos excesivamente, de igual modo sucede con el resto de relaciones establecidas anteriormente

Es decir, tras establecer la correlación entre los parámetros reabsorción ósea marginal y calidad ósea periimplantaria medidos a los 3 y 5 años en 139 localizaciones se demuestra que en nuestro estudio: Para implantes con valores altos de calidad ósea a los 3 años se produce menor reabsorción a los 5 , y precisamente aquellos que a los 5 años tienen una mejor calidad son los que menos reabsorción han sufrido a los 3. Por otra parte, a los 5 años los implantes con mejor calidad ósea y menor reabsorción son los mismos. Finalmente, tanto a los 3 como a los 5 años la menor pérdida ósea se da en los mismos implantes. Todo ello es igualmente válido si el parámetro analizado es la reabsorción que se produce en el hueso situado entre las fijaciones y no aquel que se encuentra en torno a las mismas.

\begin{tabular}{|c|c|c|c|}
\hline & Reabsorción & Reab. entre & Calidad (*) \\
\hline 3 años & $\begin{array}{c}\text { m 0,077 } \\
(\mathrm{sd} \mathrm{0,121)}\end{array}$ & $\begin{array}{c}\mathrm{m} 0,04 \\
(\mathrm{sd} \mathrm{0,099)}\end{array}$ & $\begin{array}{c}\mathrm{m} 1,390 \\
(\mathrm{sd} \mathrm{0,677)}\end{array}$ \\
\hline 5 años & $\begin{array}{c}\mathrm{m} 0,106 \\
(\mathrm{sd} \mathrm{0,128)}\end{array}$ & $\begin{array}{c}\mathrm{m} \mathrm{0,111} \\
(\mathrm{sd} \mathrm{0,172)}\end{array}$ & $\begin{array}{c}\mathrm{m} 1,839 \\
(\mathrm{sd} \mathrm{0,820)}\end{array}$ \\
\hline
\end{tabular}

(*) Calidad mínima $=0 /$ máxima $=3$

\section{DISCUSIÓN}

\section{Reabsorción ósea marginal}

El estudio radiológico se realizó según el método ya descrito por Strid o Hollender ( 4,7) y seguido por otros autores (6). Si bien es cierto que en numerosos estudios, el método radiográfico de exploración con paralelizador y cilindro largo fue apoyado con el análisis computerizado de los registros obtenidos, mientras que en el presente no se pudo disponer de esa posibilidad, creemos que el método de análisis de las imágenes realizado por tres investigadores diferentes tal y como se describe en la metodología resulta válido. Tengamos en cuenta que el estudio de la Universidad de Toronto (11) fue realizado por dos investigadores que posteriormente podían re-evaluar sus observaciones radiográficas en función de las medidas obtenidas por el otro observador. La validez de este estudio que ya puede considerarse como clásico, es reconocida por la mayor parte de la sociedad científica implantológica. Muy parecido al método establecido en nuestro estudio es el utilizado por Ahlqvist (12) en el que dos observadores realizan las medidas, obteniéndose la media en el caso de que la discrepancia sea menor o igual a $0,4 \mathrm{~mm}$. y en el caso de ser mayor se revisa de nuevo por ambos a la vez para llegar a un consenso. También Lindqvist está de acuerdo con este método de observación y así lo defiende en sus estudios (13) Hay estudios sin embargo como el de Caulier que afirman que existe una discrepancia entre la medición radiológica y la pérdida real de aproximadamente $0,85 \mathrm{~mm}$ (14). Por otra parte, estudios de Hollender y Rockler (5) aseguran que la diferencia que puede existir entre una medición radiográfica del margen óseo y el estado real de reabsorción no es mayor de 0,3mm.. Pues bien, a pesar de esta controversia, en nuestro estudio posiblemente sea la valoración de la función de anclaje de los implantes a los 3 y 5 años la que haya ofrecido unos resultados más significativos tanto clínica como estadísticamente. Es por ello que confiamos en este método como exploración rutinaria en la clínica diaria donde generalmente se carece de digitalización de la imagen, ecógrafos y otras tecnologías aplicadas al diagnóstico en implantología. No podemos por otra parte estar de acuerdo con la metodología que utiliza la radiografía panorámica para medir la reabsorción ósea tal y como utilizan otros estudios (15).

Los valores de pérdida ósea marginal en torno a los implantes son detallados por la estadística descriptiva, pero si establecemos la media de dicha reabsorción en las mediciones realizadas tanto a los 3 como a los 5 años y lo comparamos con los resultados obtenidos por otros grupos de trabajo comprobamos que los datos resultan bastante favorables como puede comprobarse en la siguiente tabla:

\begin{tabular}{|c|c|c|}
\hline Estudio & Pérdida 3 años & Pérdida 5 años \\
\hline Nuestro & $0,7 \mathrm{~mm}$. & $1 \mathrm{~mm}$. \\
\hline Jemt (6) & $1,1 \mathrm{~mm}$. & $1,2 \mathrm{~mm}$. \\
\hline Adell (2) & $1,7 \mathrm{~mm}$. & $1,9 \mathrm{~mm}$. \\
\hline Strid (7) & $1,4 \mathrm{~mm}$. & $1,6 \mathrm{~mm}$. \\
\hline Chaytor (11) & $1,6 \mathrm{~mm}$. & $1,9 \mathrm{~mm}$. \\
\hline
\end{tabular}

\section{Calidad ósea periimplantaria}

Respecto a otro de los patrones radiológicos estudiados, la calidad ósea periimplantaria ha sido explorada directamente en las radiografias periapicales ya que en ellas se puede apreciar la aparición de una fina capa de hueso cortical de cerca de $1 \mathrm{~mm}$. de espesor recubriendo en parte el implante (fig.5). 


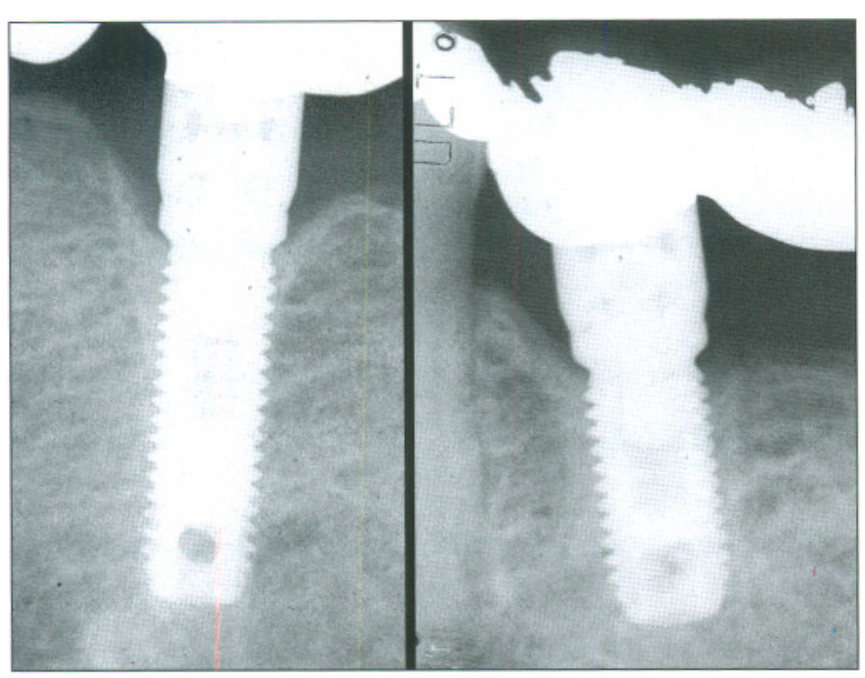

Figura 5.

Durante los controles radiográficos se observa que esta zona se va remodelando a través de los años, y tal y como relata Strid (7), dichos cambios se corresponden con una variación cuantitativa de la condensación ósea al realizarse un estudio computerizado.

Por otra parte, podemos afirmar que una de las correlaciones más interesantes que encontramos en nuestro estudio es aquella que se produce entre la reabsorción marginal y la calidad del hueso que rodea cada una de las fijaciones. Si bien comprobamos que en el primer control efectuado a los 3 años no se establece ninguna relación, cuando comparamos los resultados con los obtenidos a los 5 años, advertimos que aquellos implantes que en la primera revisión presentaban una mejor calidad ósea son los que menor reabsorción sufrirán durante los años siguientes de función. Parece por tanto que la formación de una lámina dura en torno a los implantes o lo que es lo mismo, una mayor densidad y calidad ósea son garantías para una menor reabsorción a medio plazo y por tanto una mejor predictibilidad en cuanto a la función de anclaje. También se comprueba que los implantes que a los 3 años tenían menor reabsorción son los que a los 5 demostrarán una mejor calidad periimplantaria. Este hecho parece indicar que si bien en el primer control tal vez no se ha producido todavía una perfecta reorganización trabecular en torno a las fijaciones, o no es posible advertirla con claridad en los controles radiográficos de rutina, la mejor calidad ósea en torno a algunos implantes ya comienza a manifestarse con un menor grado de reabsorción desde el inicio de la función protésica. Finalmente, se demuestra estadísticamente, que a los 5 años todos aquellos implantes con mejor calidad ósea son los que menos reabsorción han sufrido y que esta tendencia comienza ya a manifestarse en el primer control realizado a los 3 años. En todo caso, de ninguna forma según nuestro

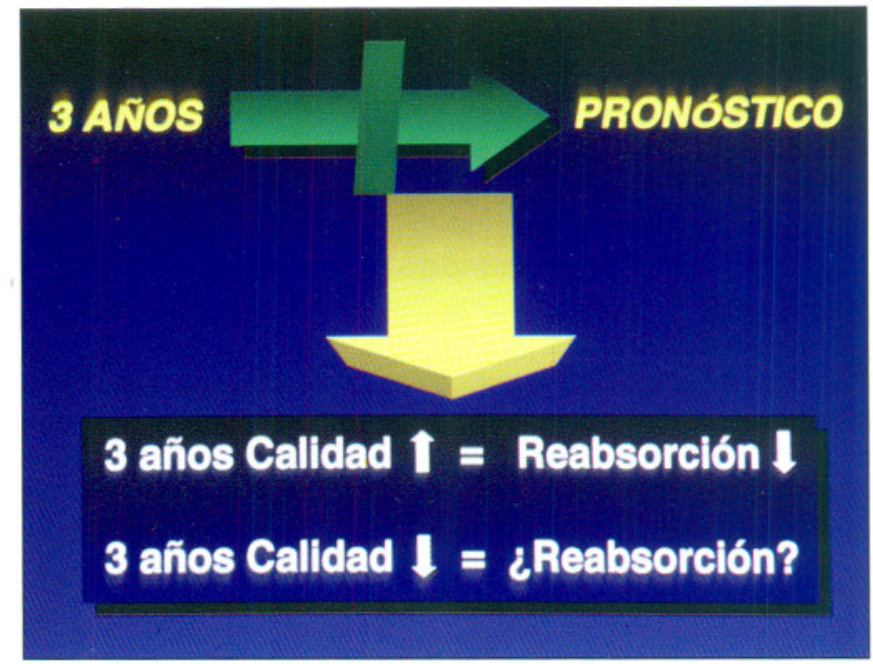

Figura 6.

estudio sería posible predecir el comportamiento óseo en torno a las fijaciones mediante un único control radiográfico a los 3 años. Sólo podríamos aventurar que aquellos implantes que en sus fases más tempranas de función presentan ya una trabeculación ósea periimplantaria consolidada, tienen un pronóstico favorable, si bien la ausencia de esta observación no indica lo contrario (Fig.6).

Según Adell y cols., existe una correlación directa entre la reabsorción marginal media en torno a los implantes y la reabsorción que se produce entre los mismos, de forma que éste podría ser otro método para comprobar la pérdida de anclaje óseo. En nuestro estudio esta teoría se corrobora y tanto en los controles realizados a los 3 como a los 5 años los valores de pérdida entre las fijaciones se correlacionan a través de una ecuación lineal con los valores de pérdida marginal de cada implante medido individualmente. Asimismo, todas las relaciones que, como comentábamos anteriormente, se demostraban entre las reabsorciones marginales medidas durante el primer y segundo control y la calidad ósea periimplantaria se confirman también cuando el parámetro de pérdida ósea es medido entre las fijaciones. Es decir, también existe una relación entre la calidad del hueso que rodea a dos implantes y el grado de reabsorción que sufre el hueso situado entre ambos. Cuanto mayor sea la primera, menor será la pérdida en esta localización.

\section{CONCLUSIONES}

Si hacemos un breve resumen de todas aquellas intenciones previas a la realización de este trabajo, podemos comenzar ratificando nuestra confianza en el 
método de seguimiento establecido, no sólo porque numerosos estudios de prestigiosos autores así lo avalen, sino porque los resultados obtenidos resultan satisfactoriamente consecuentes tanto desde el punto de vista clínico como estadístico, y por lo tanto la sencillez del método empleado creemos que lo hace recomendable como método de rutina en la clínica diaria.

Hemos comprobado que puede existir una relación bastante íntima entre la calidad del hueso que rodea todo el implante y el grado de reabsorción que se produzca en el mismo, de forma que la aparición de signos que evidencien buena calidad en los primeros controles, puede indicar un buen pronóstico en controles a más largo plazo.

No podemos, según lo registrado en la presente muestra, apoyar la hipótesis inicialmente propuesta, que decía que, si se comprobaba que a los 3 años el implante cumplía los criterios de éxito, posiblemente no sería necesaria su constatación a los 5 , debido a que ya se habría alcanzado la estabilidad de los parámetros medidos. Hemos comprobado tanto clínica como radiológicamente, que entre las revisiones realizadas a los 3 y 5 años existen en algunos casos variaciones importantes tanto en la pérdida ósea marginal como en la calidad del tejido de soporte periimplantario. Seguimos apoyando por tanto el período mínimo de 5 años aceptado generalmente y creemos que sin un seguimiento longitudinal a más largo plazo resultará imposible hecer pronósticos certeros y establecer los parámetros de seguimiento ideales

\section{ABSTRACT}

OBJETIVE: We try to evaluate if there statistical relation betwen bone mrginal resorption and bone quality around Branemark implants afther 3 and 5 years of prosthodontic function.

MATERIALS AND METHODS: We have controlled 139 Branemark implants from fixed bridges treated in the Implantology Service of The Dentistry Faculty of Madrid. Sistematic radiological exams were made in a 3 and 5 year controls.

RESULTS:Values obtained demonstrates there is no relation between bone quality and marginal resorption after 3 years function, but we obtained less resorption after 5 years in those implantsthat presented a better bone quality in the 3 years control. Those fixtures with less marginal resorption in the first period achieve a better bone quality in the second one. Finally, minor values in the bone resorption are in direct relation in the 3 and 5 years control.

CONCLUSIONS: In our sample, a statiscal relation bet- ween both studied parameters is evident, but it does not permit us to make long term predictions from observed results after three years. Although we can say that good bone quality observed during the first revisions seems to guarantee less reabsorptions in the future, we cannot assure that less quality will result in a failed treatment.

\section{KEY WORDS}

Periimplant bone resorption; periimplant bone quality

\section{BIBLIOGRAFÍA}

1. Albrektsson T, et al. The Long Term Efficacy of Currently Used Dental Implants: A Review and Proposed Criteria of Success. Int. J. Oral Maxillofac. Impl.1986;1:11-25.

2. Adell,R.,et al. A 15-year study of osseointegrated implants in the treatment of the edentulous jaw. Int. J .OralSurg. 1981;6:387-416.

3. Adell, R.Clinical results of osseointegrated implants suporting fixed protheses in edentulous jaws. J. Plast. Reconstr. Surg. 1980;14:23-46

4. Hollender L. Radiographic Examination of Endosseous Implants in the Jaws. de: Advanced Osseointegration Surgery. Worthington P. and Branemark PI. Ed Quintessence Cap.6 pag. 80-93. 1992.

5. Hollender, L. \& Rockler, B.:Radiographic evaluation of osseointegrated implants of the jaws. Dentomaxillofac. Radiol. 1980:9:91-95

6. Jemt $\mathrm{T}$ et al. Fixed implant-supported prostheses in the edentulous maxilla Clin Oral Impl Res 1994:5:142-147

7. Srtid K.G. Resultados radiográficos de: Prótesis tejidointegradas: la osteointegración en la Odontología Clínica. Branemark, Zarb y Albrektsson Edit. Quintesence 1987;Cap.11:187-198.

8. Adell R. et al. Marginal tissue reactions at osseointegrated titanium fixtures. (I). a 3-year longitudinal prospective study. Int. J. Oral Maxillofac. Surg. 1986;15:39-52.

9. Lekholm, U. et al. Marginal tisue reactions at osseointegrated titanium fixtures. (II). A cross-sectional retrospective study. Int. J. Oral Maxillofac. Surg. 1986;15:53-61. 
10. Lars w., et al .Bone resorption around fixtures in edentulous patients treated with mandibular fixed tissueintegrated prostheses. the journal of prosthettic dentistry. january 1988; 1:59-63.

11. Chaytor DV, Zarb GA, Schmitt A, Lewis DW.The longitudinal efectiveness of osseointegrated dental implants. The Toronto study: Bone level changes. Int J Per \& Restor Dentistry 1991:11:113-125.

12. Ahlqvist J, Borg K, Gunne J, Nilson H. Osseointegrated implants in edentulous jaws: a 2 year longitudinal study. Int J Oral Maxillofac Implants 1990;5:155-163.

13. Lindqvist LW, Rockler B, Carlsson GE. Bone resoption around fixtures in edentulous patients treated with mandibular fixed tissue-integrated prostheses. J of Pros Dentistry 1988:59;59-63.
14. Caulier $\mathrm{H}$, et al. The relationship of some histologic parameters, radiographic evaluations, and periotest measurements of oral implants:an experimental animal study. Int J Oral Maxillofac Implants 1997;12:380-386.

15. Leimola-Virtanen $\mathrm{R}$ et al. ITI titanium plasma-sprayed screw implants in the treatment of edentulous mandibles:a follow-up study of 39 patients. Int J Oral Maxillofac Implants 1995;10:373-378.

16. Adell R, Lekholm U, Rockler B, et al:Marginal tissue reaction at osseointegrated titanium implants.I $A$ threeyear longitudinal prospective study.Int J Oral Surg $1981 ; 15: 39-52$. 